\title{
Anemia in Diabetes - An Added Dilemma
}

\author{
Syed Ahmed Arshad and Jamal Ara
}

\section{ABSTRACT}

\begin{abstract}
Aim: To study the frequency of anemia in diabetes of an adult population.
Methodology: This cross-sectional observational study included 277 adult patients, above the age of 18 years, who were diagnosed as having diabetes by clinical and laboratory basis and were considerably controlled on their prescribed management. The study was conducted in Creek General Hospital Karachi from October to December 2020 by random based sampling technique. After a written consent answers were noted according to the questionnaire and the relevant data was tabulated and analyzed. Patients with complications or with other chronic illness, except hypertension, were excluded. The data was evaluated for the frequency of anemia in diabetics, categorizing the severity of anemia into mild, moderate, and severe. Universal variables like gender and age were assessed.

Results: Among the 277 patients in the study, there were $121(43.7 \%)$ males and $156(56.3 \%)$ females. There were $17(6 \%)$ in the young age group, 162 $(58.5 \%)$ in the middle age group and $98(35.4 \%)$ elders. The mean hemoglobin was $11.1 \mathrm{gm} / \mathrm{dl}$. Out of 277 there were $222(80 \%)$ who fell in the category of anemia. There were 90 out of $121(74 \%)$ males who were anemic and 132 out of $156(84 \%)$ females who were anemic. The most frequent category of anemia was moderate anemia $(48 \%)$ followed by mild category $(\mathbf{4 6 \%})$. Mild anemia was more frequent in males while moderate anemia was more prevalent in females. Anemia was higher in percentage in the younger group $(\mathbf{8 8 \%})$ ). Mild to moderate anemia was more prevalent than severe in all age groups. $81 \%$ of hypertensive, diabetic patients was anemic. $78 \%$ smokers had anemia. There was visible increase in frequency of anemia as the duration of diabetes increased. Conclusion: Anemia is highly prevalent in diabetics.
\end{abstract}

Keywords: diabetes, anemia, hemoglobin, hypertension.
Published Online: July 14, 2021

ISSN: $2736-5476$

DOI: 10.24018/ejclinicmed.2021.2.3.67

\section{Syed Ahmed Arshad*}

Nixor College, Pakistan

(e-mail: ahmedarshad183@gmail.com)

Jamal Ara

Creek General Hospital, Pakistan.

(e-mail: profjamalara@yahoo.com)

*Corresponding Author

\section{INTRODUCTION}

Diabetes mellitus and Hypertension are chronic diseases with worldwide increasing prevalence which have a great impact on the lives of people. The world statistics show that by the year of 2030 there will be about 440 million diabetics [1]. Hypertension is recounted to affect over 1 billion of the world's population. [2]. The increasing occurrence of diabetes mellitus is a major heath challenge for any country, especially the developing ones. The diabetic patient has a poor quality of life with being prone to consequences like blindness, renal, cardiovascular and neurological complications which affect the functional capacity of a person and eventually shortens the life span. [3]. These complications due to hypertension and diabetes are still on the upsurge in most developing countries such as Pakistan and such outcomes eventually lead to an increase in morbidity and mortality. This could be due to lack of health awareness among community, lack of approaching prompt medical management and the underdeveloped health infrastructure.
Studies show that there is a correlation between anemia and chronic illness such as diabetes and hypertension. This is partly because of the kidney damage that is a complication of these diseases but also because of another studied phenomenon. Increased or uncontrolled sugar levels cause the development of inflammatory condition with increased proinflammatory chemicals like IL-6, TNF- $\alpha$, etc. These inflammatory markers cause a decrease in the number of red blood cells which lessen the hemoglobin and cause anemia. [4].

Globally, over 1.6 billion people are reported to be anemic [2]. Anemia along with diabetes causes limitations to a healthy quality of life. It can also increase the severity of complications which then becomes an added health burden to the family and the society.

Rationale: The rationale of this study is to find the frequency of anemia in adult patients with chronic illness like diabetes at present. The results of this study will be a reminder for physicians to keep a check on the hemoglobin levels of a patient with diabetes so that anemia could be identified and corrected early in the course of disease. 


\section{MethodOlOGY}

This cross-sectional observational study included 277 adult patients, above 18 years of age, who were diagnosed as having diabetes by clinical and laboratory basis and were considered controlled on the management they were prescribed. The study was conducted in Creek General Hospital Karachi from October to December 2020 by random based sampling technique. After a written consent from the patients, their responses were logged according to the questionnaire and the relevant data was formed. Inclusion criteria included adults above age 18 years, diagnosed diabetics who are on treatment given by certified clinicians. Exclusion criteria included all patients less than 18 years, and who are not diabetic or who have an uncertain evidence of diabetes. Patients with uncontrolled diabetes, complicated or with any other chronic illness (apart from hypertension) were also not included. The data was evaluated for the frequency of anemia in patients with diabetes only, those with hypertension and diabetes. The anemia was categorized by severity of anemia into mild, moderate, and severe. Anemia frequency was analyzed in variables such as age group, gender, smokers, and duration of diabetes. The data was analyzed on SPSS.

\section{A. Definitions/Criteria}

Age group: Young age: 18-39 years, Middle age: 40-59 years, Elderly: $>60$ years.

Normal range of hemoglobin in adults: [5].

Women: Normal: $>12.0 \mathrm{~g} / \mathrm{dl}$.

Men: Normal :> 13.0g/dl.

Hemoglobin range for classification of severity of anemia into mild, moderate, and severe: [5].

Female; Mild: 11.0-11.9 g/dl, Moderate; 8.0-10.9 g/dl, Severe $:<8.0 \mathrm{~g} / \mathrm{dl}$.

Male; Mild: 11.0-12.9 g/dl, Moderate: 8.0-10.9 g/dl, Severe: $<8.0 \mathrm{~g} / \mathrm{dl}$.

Diabetes control: The sugar readings of the patient are below $120 \mathrm{mg}$ in fasting or below $200 \mathrm{mg}$ in random since the past week or recent $\mathrm{HbA} 1 \mathrm{C}$ below $7.5 \%$.

Hypertension control: Blood pressure < 130/90mmHg during the past month.

\section{RESULTS}

Out of 277 patients there were $121(43.7 \%)$ males and 156 $(56.3 \%)$ females. There were $17(6 \%)$ at the young age group, $162(58.5 \%)$ in the middle age group and $98(35.4 \%)$ elders. The mean age was 54 years. The mean hemoglobin was $11.1 \mathrm{gm} / \mathrm{dl}$ with the minimum being $4.2 \mathrm{~g} / \mathrm{dl}$ and maximum being $16.4 \mathrm{~g} / \mathrm{dl}$. 161 patients were also hypertensive.

Out of 277 there were $222(80 \%)$ who had anemia. There were 90 out of $121(74 \%)$ males who were anemic and 132 out of $156(84 \%)$ females who were anemic $(\mathrm{P}=0.034)$. The frequency of anemia in those diabetics with hypertension is 131 out of total of $161(81 \%)$ that were in the study. 32 out of $41(78 \%)$ smokers were anemic. The frequency of anemia in each severity's category is shown in Fig. 1. Gender variability in each severity of anemia is displayed in Fig. 2.

The frequency of anemia in young age group was $88 \%$ (15 out of the total of 17 that came were anemic). The middle age group were 162 in the study out of whom 130 (88\%) were anemic. Among the 98 of elder age group 77 (78\%) were in the anemic category $(\mathrm{P}=0.653)$. The severity of anemia varying with age group is shown in Fig. 3 . The correlation of anemia with duration of diabetes is tabulated in Table I.

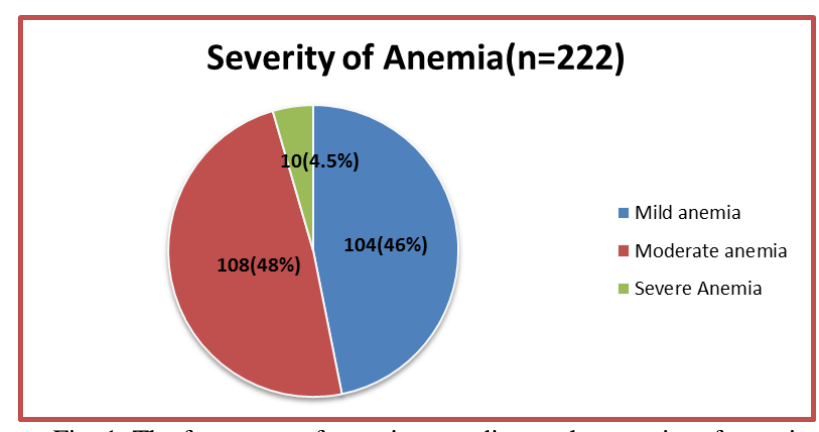

Fig. 1. The frequency of anemia according to the severity of anemia The percentages in brackets are the number in that category of anemia out of total anemic number.

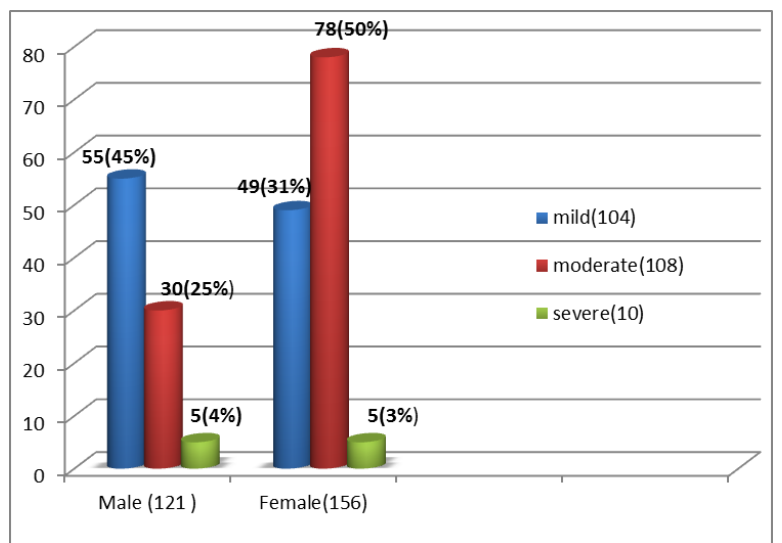

Fig. 2. The severity of anemia variability according to gender $(\mathrm{P}=000)$. The percentages in brackets are the number in the severity of anemia out of total anemic number in that gender.

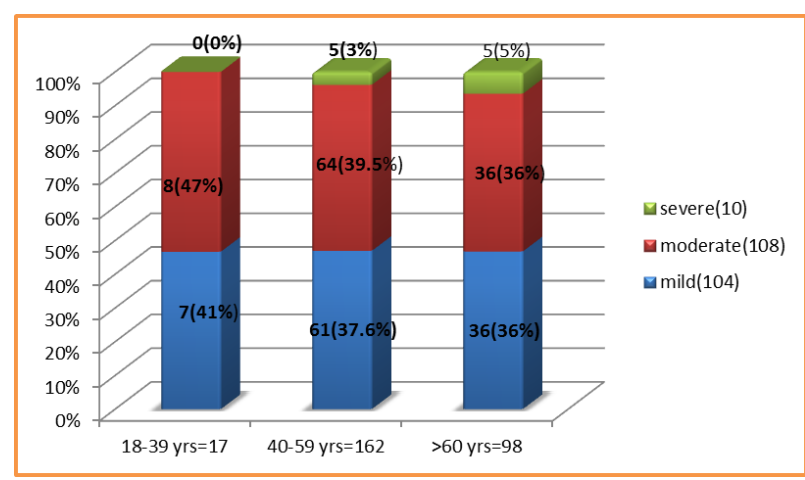

Fig. 3 The severity of anemia in each age group $(\mathrm{P}=0.866)$. The percentages in brackets are the number in the severity of anemia out of total anemic number in that age group.

TABLE I: ANEMIA CORRELATING WITH DURATION OF DiABETES $(\mathrm{P}=0.029)$

\begin{tabular}{cccc}
\hline \multirow{2}{*}{ Duration of DM } & \multicolumn{2}{c}{ Anemia present } & \\
\cline { 2 - 3 } & $\begin{array}{c}\text { yes }[\mathrm{male}<13 \mathrm{gm} / \mathrm{dl}, \\
\text { female }<12 \mathrm{gm} / \mathrm{dl}]\end{array}$ & No & \\
\hline <1 year & $8(72 \%)$ & 3 & 11 \\
1-5 years & $59(69 \%)$ & 27 & 86 \\
6-10 years & $70(85 \%)$ & 12 & 82 \\
11-20 years & $73(88 \%)$ & 10 & 83 \\
>20 years & $11(78 \%)$ & 3 & 14 \\
Total & 222 & 55 & 277 \\
\hline
\end{tabular}

*The percentage in brackets is the number of anemias out of the total number in that duration. 


\section{DISCUSSION}

The study shows a high prevalence of anemia in the diabetics of the community. This pathophysiological process is due to the internal inflammatory markers that are significantly high in chronic illness which then cause a hindrance in the production of red blood cells. The vital issue is to counteract this pathological phenomenon. The sugars and blood pressures are to be controlled and regulated to curb the inflammation but a check on the hemoglobin and its correction, if decreased, is also vital to the wellbeing of the patient.

In his study Merlin showed a significant number of people with diabetes had undiagnosed anemia and he attributed the presence of anemia to renal damage, systemic inflammation, and autonomic neuropathy [6]. Nadia [7] conducted a Pakistani study which showed $63 \%$ of diabetics to have anemia while an international study [8] showed a lower but substantial number. Our statistics showed a further rise in the frequency of anemia among diabetics over time. The mean hemoglobin in this research being lower than what it was in other studies [7] pointed that the issue of anemia has not been solved and despite several efforts the problem still lies. Numerous factors contribute to this dilemma which includes the tendency of eating junk, unhealthy food by all communities, lack of physical exercise, the decline in consumption of fresh food, use of unnecessary and excessive medications leading to gastritis, use of recreational drugs/substances, delay in obtaining healthcare due to lack of priority or poverty. All these factors directly or indirectly contribute to ill health.

The females are suffering from anemia more compared to males because of greater physiological requirements, lesser emphasis and precedence to a balanced diet and a delayed approach to healthcare facilities. This was agreed by a 2018 study in Pakistan. [9] Females here especially of less privileged class are rarely provided a complete and their required diet with early marriages and multiple pregnancies adding to the deficiencies. An Ethiopian study on diabetics found that males were more anemic than females in their area. [10].

Most studies showed elderly to have an increase in anemia frequency probably because of long duration of chronic illness, poor appetite, gastric problems, deficient diet etc. [9], [10]. Our study had different outcomes perhaps because the recent trend of eating fad diet, use of recreational substances and the surge of gastrointestinal diseases which has increased in the younger age group.

Hypertension with diabetes is an added effect to the proinflammatory pathological phenomenon and hence is more prone to anemic condition. In international studies [11], [12] the percentage of anemia was lower in hypertensive patients compared to our study, but the contributing factors are still there which need to be addressed such as an adoption of sedentary lifestyle, a high salt diet, unbalanced diet, regular medical checkups.

The severity of anemia was mild to moderate in most studies [13], including ours. A mild severity of anemia can be corrected if it is checked early, and the risk factors are studied and erased.

This proportional trend of increased anemia frequency as the diabetic duration is prolonged is followed around the globe understandably due to the increase in the inflammation process with the development of complications and resistance to the diabetic control [13].

The research proves that much is needed to be done to create awareness of sugar and blood pressure control, to convince people to get early and regular follow-ups/checkups and to consume a balanced diet with physical activity. It is only then that we can become a healthy proactive nation.

\section{CONCLUSION}

Anemia has significantly increased in diabetics especially in females, younger age group and those with hypertension.

\section{REFERENCES}

[1] J. E. Shaw, R. A. Sicree, and P. Z. Zimmet. "Global estimates of the prevalence of diabetes for 2010 and 2030," Diabetes Research and Clinical Practice. 2010: 87(1): 4-14.

[2] Anderson AK. Prevalence of Anemia, Overweight/Obesity, and Undiagnosed Hypertension and Diabetes among Residents of Selected Communities in Ghana. Int J Chronic Dis. 2017; 2017:7836019. doi: $10.1155 / 2017 / 7836019$.

[3] P. M. S. B. Francisco, A. P. Belon, M. B. A. Barros, L. Carandina, M. C. G. P. Alves, and C. L. G. Cesar. "Self-reported diabetes in the elderly: prevalence, associated factors, and control practices," Cadernos de Saúde Pública. 2010: 26(1):175-184.

[4] A. Angelousi and E. Larger, "Anaemia, a common but often unrecognized risk in diabetic patients: a review," Diabetes \& Metabolism, vol. 41, no. 1, pp. 18-27, 2015.

[5] Haemoglobin concentrations for the diagnosis of anaemia and assessment of severity. https://www.who.int/vmnis/indicators/haemoglobin.pdf.

[6] Merlin C.T., Richard J. M., Con T., David P., George J. Unrecognized Anemia in Patients with Diabetes. A cross-sectional survey. Anemia in diabetes; in age group and gender. Diabetes Care. 2003 Apr; 26(4): 1164-1169. https://doi.org/10.2337/diacare.26.4.1164.

[7] Nadia S., Osman M.H. Newly Diagnosed Anemia in Admitted Diabetics, Frequency, Etiology and Associated Factors. JCPSP. 2015: 25 (4): 242-246.

[8] Barbieri J, Fontela PC, Winkelmann ER, Zimmermann CE, Sandri YP, Mallet EK, Frizzo MN. Anemia in Patients with Type 2 Diabetes Mellitus. Anemia. 2015; 2015: 354737. doi: 10.1155/2015/354737. Epub 2015 Nov 11. PMID: 26640706; PMCID: PMC4658398.

[9] Salma M. AlDallala, c, Nirupama Jenab. Prevalence of Anemia in Type 2 Diabetic Patients. Journal of Hematology. 2018. (7) 2; 57-61.

[10] Fiseha T, Adamu A, Tesfaye M, Gebreweld A (2019) Prevalence of anemia in diabetic adult outpatients in Northeast Ethiopia. PLoS ONE 14(9): e0222111. https://doi.org/10.1371/journal.pone.0222111.

[11] Paul B, Wilfred NC, Woodman R, Depasquale C. Prevalence and correlates of anaemia in essential hypertension. Clin Exp Pharmacol Physiol. 2008 Dec; 35(12): 1461-4. doi: 10.1111/j.14401681.2008.05031.x. Epub 2008 Aug 26. PMID: 18759858.

[12] Kim-Mitsuyama, S., Soejima, H., Yasuda, O. et al. Anemia is an independent risk factor for cardiovascular and renal events in hypertensive outpatients with well-controlled blood pressure: a subgroup analysis of the ATTEMPT-CVD randomized trial. Hypertens Res 42, 883-891, (2019). https://doi.org/10.1038/s41440-019-0210-1.

[13] Taderegew MM, Gebremariam T, Tareke AA, Woldeamanuel GG. Anemia and Its Associated Factors Among Type 2 Diabetes Mellitus Patients Attending Debre Berhan Referral Hospital, North-East Ethiopia: A Cross-Sectional Study. J Blood Med. 2020; 11: 47-58. https://doi.org/10.2147/JBM.S24323.

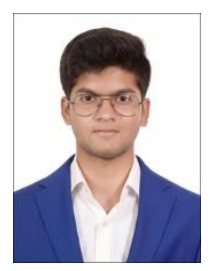

Syed Ahmed Arshad is an A-level student of Nixor College, Karachi, Pakistan, of Cambridge GCE University. Inspired to pursue a medical degree, Syed Ahmed found himself doing an internship under Dr. Prof. Jamal Ara, the head of department of Medicine at Creek General Hospital, Karachi. Under her supervision and guidance, Syed Ahmed conducted this research. Furthermore, he has previously published the article 'Non-Adherence - A Challenge in Health Management' in the World Journal of Pharmaceutical and Medical Research Journal (wjpmr, 2020,6(7), 74-77). 